\title{
Negative Whole-Body Computed Tomography Scans in Polytrauma Patients: A Retrospective Cohort Study
}

This article was published in the following Dove Press journal: Open Access Emergency Medicine

\author{
Nisreen H Maghraby (D) \\ Hassan M Alshaqaq $\mathbb{D}^{2}$ \\ Abdullah Saleh AIQattan (1) ${ }^{3}$ \\ Adnan Fawzi Alfaraj $\mathbb{D D}^{4}$ \\ Omar A Alghamdi $\mathbb{D}^{2}$ \\ Malak J Alzawad ${ }^{5}$ \\ David A Farcy $\mathbb{D D}^{6}$ \\ 'Department of Emergency Medicine, \\ King Fahd University Hospital-College of \\ Medicine, Imam Abdulrahman Bin Faisal \\ University, AlKhobar, Kingdom of Saudi \\ Arabia; ${ }^{2}$ College of Medicine, Imam \\ Abdulrahman Bin Faisal University, \\ Dammam, Kingdom of Saudi Arabia; \\ ${ }^{3}$ Department of General Surgery, King \\ Fahad Specialist Hospital-Dammam, \\ Kingdom of Saudi Arabia; ${ }^{4}$ Department of \\ Emergency Medicine, King Fahad \\ Specialist Hospital-Dammam, Kingdom of \\ Saudi Arabia; ${ }^{5}$ Department of Psychiatry, \\ Ministry of Health, Dammam, Kingdom of \\ Saudi Arabia; ${ }^{6}$ Mount Sinai Medical \\ Center, Department of Emergency \\ Medicine, Miami Beach, Florida, USA
}

Correspondence: Nisreen H Maghraby King Fahd University Hospital-College of Medicine, Imam Abdulrahman Bin Faisal University, 6844 King Faisal University City, Dammam 3026-342II, Saudi Arabia Tel +966504807318

Email nhmaghraby@iau.edu.sa
Background: Whole-body computed tomography (WBCT) has been a mainstay and an integral part of the evaluation of polytrauma patients in trauma centers and emergency departments (ED) for a comprehensive evaluation of the extent of injuries. However, routine use of WBCT remains controversial since it exposes patients to radiation and exponentially increases financial expense. The primary objective was to determine the rate of negative WBCT in polytrauma patients.

Patients and Methods: A retrospective cohort study was conducted at an academic hospital in the Kingdom of Saudi Arabia, which is a dedicated trauma center with a mean of 237,392 ED visits and 10,714 trauma per year. The study included all adult ( $\geq 18$ years) polytrauma patients who presented to our ED, requiring trauma team activation, and underwent WBCT as part of their evaluation from January 2016 to May 2017. We excluded pediatric patients, patients transferred from another facility, and pregnant patients. The primary endpoint was to measure the rate of negative WBCT in polytraumatized patients.

Results: A total of 186 patients were included with a mean age of $28.8 \pm 12.9$ years. The rate of negative WBCT scans was $20.4 \%$. The positive scans were subclassified based on the number of anatomical body regions that were affected radiologically. One body region was affected in 47 patients (31.8\%), two body regions were affected in 50 patients $(33.8 \%)$, and $\geq 3$ body regions were affected in 51 patients $(34.3 \%)$. In a subset analysis, we identified that oxygen saturation $<94 \%$ and GCS $\leq 8$ were associated with positive CT scans.

Conclusion: Our study revealed a slightly higher rate of utilization of WBCT in the management of trauma patients compared to studies with similar practice. We believe that in the correct setting with incorporating high index of suspicion, a physical examination with attention to vital signs and mental status, performing E-FAST, and dedicated X-Rays is a way to potentially reduce the use of WBCT in polytrauma patients.

Keywords: negative rate, whole-body, selective, computed tomography, multiple, polytrauma, MVCs

\section{Key Messages}

What is already known on this subject:

- WBCT has become the preferred imaging modality for evaluating polytrauma patients due to its rapid and highly sensitive detection of acute traumatic injuries.

- WBCT has demonstrated statistical and clinical significance, in multiple observational studies, in terms of decreasing mortality rate and better outcome. 
- Although multiple studies reported different algorithms and criteria to limit unnecessary WBCT in polytraumatized patients; yet, the choice either to perform WBCT or selective imaging is still a matter of debate.

What this study adds

- The rate of negative WBCT in the current study was slightly higher than the average reported percentages in centers with similar indications to request WBCT.

- ISS scores $\geq 24$, GCS $\leq 8$, and oxygen saturation $<94 \%$ demonstrated a statistically significant association with positive CT scans.

- This study demonstrates the need for appropriate selection criteria, which might reduce unnecessary WBCT scans safely.

\section{Introduction}

Trauma remains one of the main causes of morbidity and mortality worldwide, and it is considered the number one cause of death in patients under 45 years of age. ${ }^{1}$ In the Kingdom of Saudi Arabia (KSA), motor vehicle collision (MVC) is the most common mechanism of injury in trauma patients, accounting for $80-85 \%$, with a $4.7 \%$ mortality rate. Hence, this is a well-known socioeconomic burden on multiple levels, including patients, their families, and the healthcare system. ${ }^{2}$ The Saudi ministry of health hospitals reported that $80 \%$ of their deaths are attributed to MVC, and these victims occupied one-fifth of the hospital beds in a consistent manner. ${ }^{3}$

It is crucial to rapidly detect injuries and provide definitive treatment in critically injured polytrauma patients to achieve optimal care and outcomes. ${ }^{4}$ The advances in whole-body computed tomography (WBCT) have integrated its use in the diagnostic approach as a standard practice among polytrauma patients in trauma centers and emergency departments for a comprehensive evaluation of the extent of injuries, owing to its high sensitivity in detecting trauma-related injuries promptly. ${ }^{5}$ Moreover, WBCT has been associated with a lower rate of missed injuries, prompt definitive management, quick disposition, and decreased mortality rate. ${ }^{6}$

In contrast, WBCT limitations include increased radiation exposure and possible anaphylaxis. Until recently, contrast-induced nephropathy was considered as one of the limitations that is related to WBCT scans. However, recent studies have demonstrated no difference between contrast enhanced CT and non-contrast CT scans in increasing the frequency of post-radiocontrast acute kidney injury. ${ }^{7,8}$ As a result of the mentioned limitations, routine use of WBCT remains controversial. As an alternative, a selective $\mathrm{CT}$ imaging approach could potentially replace the unnecessary WBCT scans. The group of patients who benefit from WBCT has been questioned in the reported literature. ${ }^{6}$ Accordingly, further high-quality research should be conducted to optimize the current suggested criteria reported in the literature. ${ }^{6,9-14}$ The primary objective of this study was to determine the rate of negative WBCT in patients involved in polytrauma.

\section{Patients and Methods}

This study was approved by the Institutional Review Board (IRB), in which informed consent was waived. It is reported in accordance to the STROBE reporting check list, institutional and national ethics committees, and with the Helsinki Declaration (as revised in 2013).

\section{Study Design}

We performed a retrospective cohort study that included all polytrauma patients who presented to the emergency department (ED) of King Fahad University Hospital, which is an academic hospital in the Eastern Province of the KSA. It is a dedicated trauma center with a mean of 237,392 ED visits and 10,714 trauma per year. Patients who underwent trauma team activation were recorded prospectively in a database as a part of our institutional policy. In this database, standardized trauma sheets were filled for every patient prospectively, which include demographic variables, mechanism of injury, vital signs, physical examination, investigations, ED intervention, and ED disposition. The study recruitment took place over 16 months, between the period from January 2016 to May 2017. Polytrauma (also known as multiple trauma) was defined as a trauma that involves two or more body regions. All adult (age $\geq 18$ years) patients who presented with polytrauma whose condition required trauma team activation and that underwent WBCT as a part of their initial ED evaluation were included. We excluded any pediatric patients under 18 years of age, patients transferred from another facility, pregnant patients, patients who died before WBCT, and patients who did not have WBCT as part of their initial evaluation (post-intervention). 


\section{Initial Trauma Management}

The involvement of the trauma team is a two-tiered protocol, which is either trauma team activation or trauma team consult. The ED team leader makes the decision to activate the trauma team upon patient arrival, which is a physician decision that is based on the hospital's trauma activation protocol. This decision is based on several variables, including mechanism of injury, vital signs, and primary survey findings. Moreover, whether to do a WBCT scan or not is based on the trauma team leader's clinical judgment relying crucially on a severe mechanism of injury. Specifically, ejection from a vehicle, death or severe injury of an occupant in the same collision, highspeed crash, rollover crash, fall from a height ( $>6$ meters), no seatbelt, and no helmet protection. The judgment of the trauma team leader also takes into consideration several factors, including clinical injuries to more than two regions, suspicion of serious injuries, evidence of intoxication, compromised vital signs, suspected spinal cord injury, unconscious patients, intubated patients, sedated patients, and unreliable physical examination. However, there is no established and validated pre-specified criteria for selection, where all the above mentioned are according to individual clinical judgement, which might be dominated by subjectivity. But having severe mechanism of injury, combined with polytrauma, either clinically or radiologically evident, was generally considered reasonable to request a WBCT. In our hospital, emergency medicine attendings are in charge as trauma team leaders until the arrival of the trauma team; then, trauma surgery attending will take over. There was no change or variation in the trauma or imaging protocols throughout the study period. Trauma team activation in our institute consisted of trauma surgery, orthopedic surgery, neurosurgery, anesthesiology, and respiratory therapy services. However, vascular, thoracic, and plastic services participate when explicitly called.

\section{Imaging Protocol}

The institutional standard WBCT protocol is a noncontrast head and neck, followed by a contrast-enhanced arterial phase of chest, abdomen, and pelvis, then a contrast-enhanced venous phase for abdomen and pelvis. It extends from the vertex to the ischial tuberosities. All the scans were performed by SOMATOM Definition Flash 128 Slices Dual-Source CT Scanner (Siemens, Munich, Germany). Furthermore, the contrast material was injected using a MEDRAD Stellant automated CT injector $\left(\right.$ MEDRAD $^{\circledR}$, USA) at a dose of $(1.5-2) \mathrm{cc} / \mathrm{kg}$ to the forearm veins. The contrast material used in our center is OMNIPAQUE 300 (Guerbet $^{\circledR}$, Villepinte, France). WBCT reports were written by the on-call radiologist and verified by the radiologist attending consultant. All data presented in this study was obtained following the verification of the report by the radiology consultant. The reports were classified into the following regions: head, spine, chest, abdomen, and pelvis. The results of WBCT reports were valued as positive or negative, with further subclassification of the positive WBCT to describe the pattern and injuries related to each region. The positive scan was defined as any acute traumatic injury recognized by the radiologist in any single region from vertex to pelvis. In contrast, a negative scan was defined as the absence of acute traumatic injury in all regions from head to pelvis.

\section{Statistical Analysis}

The analysis was performed on 186 patients after the application of the inclusion and exclusion criteria. The primary analysis was measuring the rate of negative WBCT in patients involved in polytrauma. On the other hand, the secondary analysis was to investigate if a set of initial findings were correlated with positive CT scans. All the statistical analysis was performed by Statistical Packages for Social Sciences (SPSS) version 20 (IBM, Armonk, NY, USA). A P-value of less than or equal to 0.05 deemed to be statistically significant. Missing values were excluded. All the categorical variables were presented as frequencies and percentages, and the continuous variables were presented as mean and standard deviation (SD) for the normally distributed variables, whereas non-normally distributed variables were presented as median with inter-quartile range (IQR). Normality was tested using the Shapiro-Wilk test. A Chisquare test was conducted to compare two categorical variables. Univariate regression analysis of the secondary outcomes had been undertaken to predict the negative CT scans among the different regions of the body, where the odds ratio and $95 \%$ confidence interval (CI) were also reported.

\section{Results}

\section{Descriptive Analysis}

A total of 186 patients were included in our study. The flow chart is summarized in Figure 1. Among the 186 


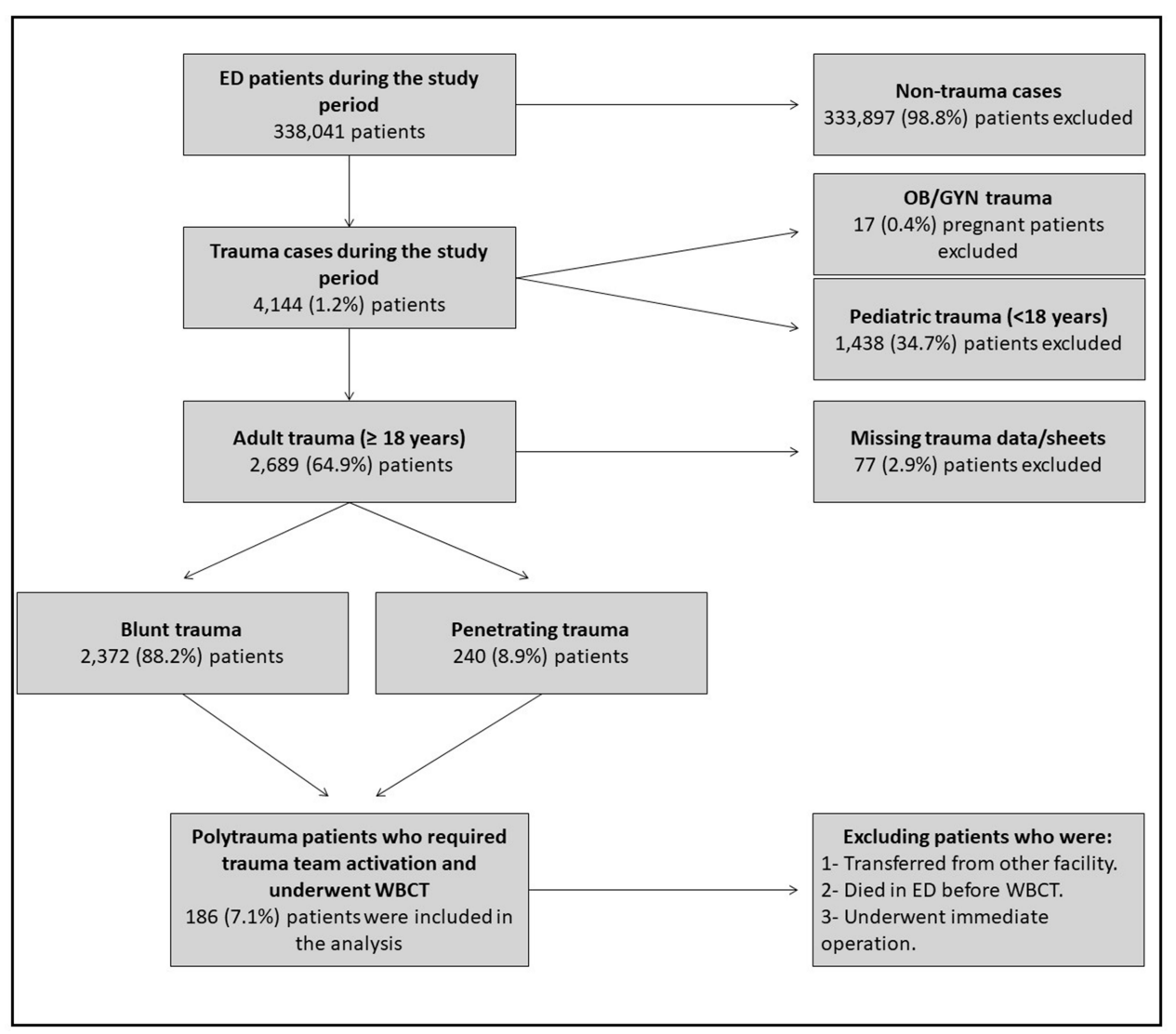

Figure I Flow chart of the recruitment process. ED, emergency department; OB/GYN, Obstetric and Gynecology; WBCT, whole-body computed tomography.

patients, $156(83.9 \%)$ were males, and $30(16.1 \%)$ were females. The mean age of patients was $28.8 \pm 12.9$ years old. Most of the study population were blunt trauma patients accounting for $87.6 \%$; on the other hand, penetrating trauma accounted for $12.4 \%$. The most common mechanism of injury was MVC (73.5\%), followed by stab wound $(9.2 \%)$, fall from height $(8.6 \%)$, pedestrian injury $(5.4 \%)$, assault injuries $(2.2 \%)$, and gunshot wound (1.1\%), respectively. From the MVC category, 16 patients $(11.8 \%)$ were ejected from the vehicle.

The mean of the initial systolic blood pressure (SBP) was $121 \pm 23$, and the mean arterial pressure (MAP) was $87.4 \pm 17.3$. The initial mean pulse rate was $99.4 \pm 24.9$, the mean respiratory rate was $21.4 \pm 4.0$, and the oxygen saturation $\left(\mathrm{SpO}_{2}\right)$ was $96.4 \pm 6.4 \%$. ED disposition showed that 63 patients $(33.9 \%)$ were admitted to the surgical intensive care unit (SICU), 57 patients (30.6\%) immediately went to the operating theatre, 51 patients (27.4\%) were admitted in the ward, and 15 patients (8.1\%) were discharged after being observed and cleared in the ED. The mean hospital length of stay (HLOS) was $21.7 \pm 36$ days. Subclassifying the duration of the SICU group showed 121 patients $(65.1 \%)$ had a LOS in the SICU of $1-3$ days, 20 patients (10.7\%) had 4-6 days, and 45 patients $(24.2 \%)$ had more than six days of stay in the SICU. The descriptive data are summarized in Table 1.

The mean Glasgow Coma Score (GCS) was $12 \pm 4$; when we further subclassified the scores it showed 115 patients $(61.8 \%)$ were in the mild category (13-15 score range), 22 patients (11.8\%) were in the moderate category (9-12 score range), and 49 patients $(26.3 \%)$ were in the severe category (3-8 score range). The median injury severity score (ISS) was 25 (16-33.5), and further subclassification showed 10 patients $(5.4 \%)$ had ISS in the scale of $1-8$, 32 patients $(17.3 \%)$ had ISS score in the scale of 9-15, 49 patients $(26.5 \%)$ had ISS score in the scale of 16-24, and 94 patients $(50.8 \%)$ had ISS score more than 24 . We did not perform comorbidities exclusion because the patients' age base was young.

\section{Outcome Analysis}

The rate of negative WBCT scans was 20.4\% (38 patients), while the positive rate was $79.6 \%$ (148 patients). The positive 
Table I Description of Demographic Data

\begin{tabular}{|c|c|}
\hline Study Variables & $N(\%)(n=186)$ \\
\hline Age, years $^{\mathrm{a}}$ & $28.8 \pm 12.9$ \\
\hline $\begin{array}{l}\text { Gender } \\
\text { - Male } \\
\text { - } \text { Female }^{\mathrm{b}}\end{array}$ & $\begin{array}{l}156(83.9 \%) \\
30(16.1 \%)\end{array}$ \\
\hline \multicolumn{2}{|l|}{ Mechanism of Injury* } \\
\hline $\begin{array}{l}\text { Blunt }^{\mathrm{b}} \\
\text { - } \text { MVC }^{\mathrm{b}} \\
\text { - Fall from height } \\
\text { - Pedestrian Injury }\end{array}$ & $\begin{array}{l}162(87.6 \%) \\
136(73.5 \%) \\
16(8.6 \%) \\
10(5.4 \%)\end{array}$ \\
\hline $\begin{array}{l}\text { Penetrating }^{\mathrm{b}} \\
\text { - Stab wound } \\
\text { - Gunshot wound } \\
\text { - Assault }\end{array}$ & $\begin{array}{l}23(12.4 \%) \\
17(9.2 \%) \\
2(1.1 \%) \\
4(2.2 \%)\end{array}$ \\
\hline $\begin{array}{l}\text { Pulse (beats per minute })^{\mathrm{a}} \\
\text { MAP }(\mathrm{mm} \mathrm{Hg})^{\mathrm{a}} \\
\text { SBP }(\mathrm{mm} \mathrm{Hg})^{\mathrm{a}} \\
\text { RR (breaths per minute) }{ }^{\mathrm{a}}\end{array}$ & $\begin{array}{l}99.4 \pm 24.9 \\
87.4 \pm 17.3 \\
121 \pm 23 \\
21.4 \pm 4.0\end{array}$ \\
\hline $\begin{array}{l}\text { GCS } \text { (points) }^{\mathrm{a}} \\
\text { - Mild (13-15 score range) } \\
\text { - Moderate }(9-15 \text { score range })^{\mathrm{b}} \\
\text { - Severe (3-8 score range) }\end{array}$ & $\begin{array}{l}12 \pm 4 \\
115(61.8 \%) \\
22(11.8 \%) \\
49(26.3 \%)\end{array}$ \\
\hline $\begin{array}{l}\text { E-FAST } \\
\text { - Positive } \\
\text { - } \text { Negative }^{\mathrm{b}}\end{array}$ & $\begin{array}{l}38(20.7 \%) \\
146(79.6 \%)\end{array}$ \\
\hline $\begin{array}{l}\text { ED Disposition } \\
\text { - } \mathrm{OR}^{\mathrm{b}} \\
\text { - } \mathrm{SICU}^{\mathrm{b}} \\
\text { - Ward } \\
\text { - Discharge }\end{array}$ & $\begin{array}{l}57(30.6 \%) \\
63(33.9 \%) \\
51(27.4 \%) \\
15(8.1 \%)\end{array}$ \\
\hline 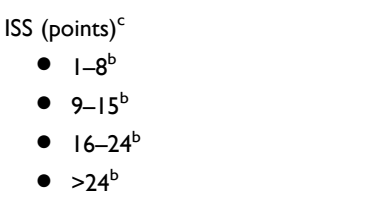 & $\begin{array}{l}25(16-33.5) \\
10(5.4 \%) \\
32(17.3 \%) \\
49(26.5 \%) \\
94(50.8 \%)\end{array}$ \\
\hline HLOS (days) ${ }^{a}$ & $21.6 \pm 36$ \\
\hline 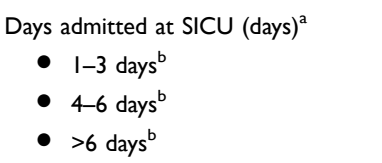 & $\begin{array}{l}4.8 \pm 7.9 \\
121(65.1 \%) \\
20(10.7 \%) \\
45(24.2 \%)\end{array}$ \\
\hline $\begin{array}{l}\text { Days admitted at Ward } *(\text { days })^{\mathrm{a}} \\
\text { - } 1-3 \text { days }^{\mathrm{b}} \\
\text { - } 4-6 \text { days }^{\mathrm{b}} \\
\text { - }>6 \text { days }\end{array}$ & $\begin{array}{l}17 \pm 32 \\
51(28 \%) \\
33(16 \%) \\
102(56 \%)\end{array}$ \\
\hline
\end{tabular}

Notes: *The missing data were excluded. Data represented as, ${ }^{a}$ mean $\pm S D$, ${ }^{\mathrm{b}}$ frequency (\%), or ${ }^{\mathrm{c}}$ median (IQR).

Abbreviations: MVC, motor vehicle collisions; MAP, mean arterial pressure; SBP, systolic blood pressure; RR, respiratory rate; GCS, Glasgow Coma Scale; E-FAST, Extended Focused Assessment with Sonography for Trauma; ED, emergency department; OR, operating room; SICU, surgical intensive care unit; ISS, Injury Severity Score; HLOS, hospital length of stay.
Table 2 Description of Body CT Scans $(n=186)$

\begin{tabular}{|l|l|l|}
\hline Study Variables & Negative N (\%) & Positive N (\%) \\
\hline WBCT & $38(20.4 \%)$ & $148(79.6 \%)$ \\
Head CT Scan & $107(57.5 \%)$ & $79(42.5 \%)$ \\
Spine CT Scan & $129(69.4 \%)$ & $57(30.6 \%)$ \\
Chest CT Scan & $79(42.5 \%)$ & $107(57.5 \%)$ \\
Abdomen CT Scan & $129(69.4 \%)$ & $57(30.6 \%)$ \\
Pelvis CT Scan & $168(90.3 \%)$ & $18(9.7 \%)$ \\
\hline
\end{tabular}

scans were further subclassified based on the number of anatomical body regions that were affected radiologically. One body region was affected in 47 patients $(31.8 \%)$, two body regions were affected in 50 patients $(33.8 \%)$, three body regions were affected in 31 patients $(20.9 \%)$, four body regions were affected in 16 patients $(10.8 \%)$, and five body regions were affected in four patients $(2.7 \%)$. Exploring the negative scans showed that there were 107/ 186 (57.5\%) negative head CT scans, 129/186 (69.4\%) negative spinal CT scans, 79/186 (42.5\%) negative chest CT scans, 129/186 (69.4\%) negative abdominal CT scans, and 168/186 (90.3\%) negative pelvis CT scans (See Table 2, Supplementary Table 1, Figure 2, and Figure 3).

As a secondary observation during the data interpretation, Pearson correlation showed a weakly negative correlation $(-0.154)$ between the negative WBCT and increased HLOS with a p-value of 0.036 . Moreover, positive WBCT showed a weakly positive correlation with the GCS score, which is demonstrated by the correlation coefficient value of 0.16 (p-value of 0.031). Positive abdomen, pelvis, and chest scans were associated with $>24$ ISS, which was statistically significant $(\mathrm{p}-<0.001)$. When univariate logistic regression analysis was performed on ISS $>24$, it demonstrated a statistically significant negative effect on the negative

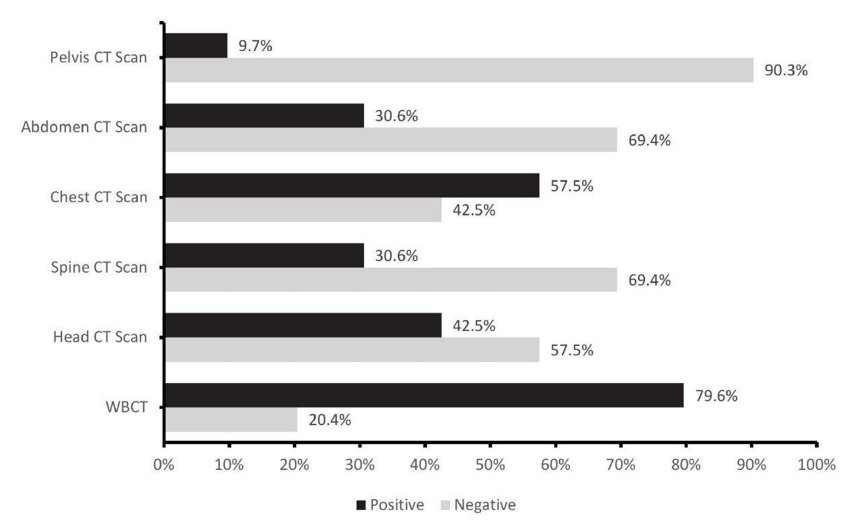

Figure 2 The rate of negative and positive CT scans. CT, Computed Tomography; WBCT, whole-body computed tomography. 


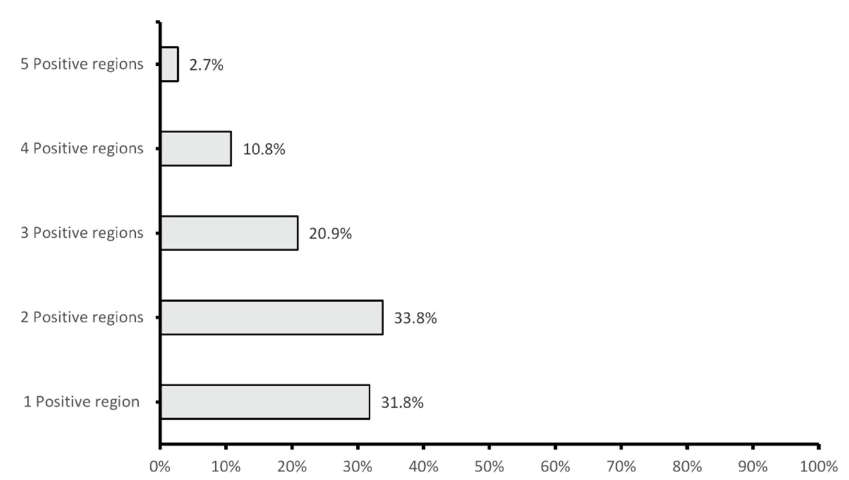

Figure 3 Description of the pattern of positive regions in the positive WBCT group. WBCT, whole-body computed tomography.

abdominal scans (odds ratio 0.343, CI [0.157-0.752], $\mathrm{p}-0.008$ ) and negative pelvic scans (odds ratio 0.207, CI [0.046-0.943], p-0.042). On the other hand, the odds ratio was not considered statistically significant for negative thoracic scans (odds ratio 0.483, CI [0.233-1.000], p-0.050).

Additionally, the patients in the severe category (3-8) of GCS showed a significant association with positive WBCT scans $(\mathrm{p}-<0.001)$ in contrast to the mild category (13-15) where it had higher negative WBCT rates; while, the moderate category (9-12) was deemed with no significant association. Furthermore, univariate logistic regression analysis showed severe GCS category (3-8) to have a statistically significant negative effect on negative head CT scans (odds ratio 0.256, CI [0.086-0.760], p-value 0.014). Regarding the chest CT scans, oxygen saturation revealed a significant association $(\mathrm{X} 2=8.228, \mathrm{p}-0.004)$, where stable ( $\geq 94 \%$ ) saturation was associated with negative scans compared to unstable saturation $(<94 \%)$. In addition, univariate logistic regression analysis showed unstable oxygen saturation $(<94 \%)$ to have a significant negative influence on the negative chest CT scans (odds ratio 0.238 , CI [0.085-0.670], p-0.006).

\section{Discussion}

The present study determined the rate of negative WBCT scans among polytrauma patients, which was $20.4 \%$. The study also found that $31.8 \%$ of the positive WBCT scans had only one positive region, supporting the authors' belief that a focused selective CT scan can potentially replace this group of scans. The study findings raise the concern that a substantial percentage of our patients might be exposed to unnecessary scans. Exposure to unnecessary scans could lead to adverse effects on several aspects, including healthcare expenses and radiation exposure. Formerly, post-radiocontrast acute kidney injury was a concern during CT decision-making, which is encountered in daily ED practice. ${ }^{7}$ However, a recent metaanalysis of observational studies that involved 107,335 patients examined the frequency of post-radiocontrast acute kidney injury which showed no association between contrast-enhanced CT with neither acute kidney injury, need for dialysis, nor increased mortality rate when compared to a control group of non-contrast CT scans. ${ }^{8}$

It is crucial to weigh the use of WBCT for each patient to safely minimize unnecessary radiation to the young trauma population. Our study identified a potential recommendation to decrease the rate of negative WBCT use by applying an appropriate patient selection criteria, but a comprehensive conclusion remains challenging. In adjacent with severe mechanism of injury, we found low GCS score (3-8) to be significantly associated with positive WBCT and head CT scans. Also, the presence of respiratory abnormality defined by $\mathrm{SpO}_{2}$ below 94\% was significantly associated with positive chest CT scans.

Comparing the rate of negative WBCT scans to other international rates showed that we have a slightly higher rate than similar studies with strict use of WBCT to severe polytrauma; however, we still occupy the middle of the range. Specifically, the range of negative WBCT starts from as low as 7\% and goes as high as 57\%. ${ }^{6,10,12,15-18}$ Locally, only one study done by Orf et al reported the rate of negative WBCT in a single institute in the KSA, which was $30.5 \%{ }^{19}$ However, this study included only the victims of MVCs.

The rate of negative $\mathrm{CT}$ scans was highest for the pelvis $(90.3 \%)$ and lowest for chest CT scans $(42.5 \%)$. The low rate of negative chest CT scans might be attributed to the low rate of seat belt usage in the KSA that resulted in a secondary collision, which occurs between the unbelted occupant chest and the vehicle steering wheel. ${ }^{20}$ Bendak has reported the average appliance of the seat belt in the KSA to be $60 \%$ for drivers and $22.7 \%$ for front-seat passengers during the study period. ${ }^{20}$

Globally, trauma practice has been integrating the use of WBCT in the evaluation of patients with multiple injuries to two or more anatomical regions simultaneously. WBCT has become the preferred imaging modality due to its rapid and highly sensitive detection of acute traumatic injuries. ${ }^{5,15}$ Although WBCT demonstrates a benefit in rapid recognition and treatment, it has been shown that only one-fifth $(\sim 19 \%)$ of the patients' management was changed by the WBCT findings. ${ }^{21}$ On the other hand, another study by Oosthuizen et al has reported $77 \%$ of 
WBCT influenced the course of management in their polytrauma cohort. ${ }^{15}$ The main difference between the two studies was the policy of ordering WBCT. The study by Salim et al used a liberal WBCT scanning policy, compared to the study by Oosthuizen et al, which applied a strict protocol for the use of WBCT in their trauma patients to minimize unnecessary scans. ${ }^{15,21}$

Our secondary observation showed that mechanism of injury, ISS score of more than 24 , GCS $\leq 8$, oxygen saturation $<94 \%$ to be statistically significant with positive CT scans; however, a clinical judgment based on a combination of multiple data points including the severe mechanism of injury, compromised vital signs, physical examination, radiographic trauma series, and E-FAST scan is essential for the selection of patients who should be undergoing WBCT. ${ }^{14}$ Likewise, the simultaneous use of other decision rules, including Canadian CT head rule, Canadian C-spine rule, and NEXUS chest CT, may aid in the decision-making process. ${ }^{1}$ Equilibrium must be taken in the selection criteria to avoid underutilizing WBCT scans, leading to missing critical injuries. Griffey et al have reported that emergency attendings agreed on the fact that CT overuse is a problem and called for decision support that could aid in solving this healthcare issue. ${ }^{22}$

It was hypothesized that negative WBCT scans might lead to early discharge that possesses several benefits, including optimization of inpatient throughput, improving patients' flow in the ED, decrease time to diagnosis, decrease time to definitive treatment, and shorten HLOS. ${ }^{1,14}$ Nonetheless, only 15 patients in our study were discharged out of the 38 patients who had completely negative WBCT. The rest of the patients were admitted either due to pain, intoxication, or social reasons.

Globally, the choice whether to perform WBCT or selective imaging is still a matter of debate. ${ }^{4,15}$ This controversy arose based on the fact that some observational studies advocated the use of selective scanning, ${ }^{23}$ while others $^{24,25}$ demonstrated the survival benefits of WBCT over selective scanning. ${ }^{10,26}$

However, the first international, multi-center, randomized controlled trial (REACT-2) compared the effect of using an immediate WBCT scanning versus conventional imaging and selective CT scanning in 1403 randomly assigned patients with severe trauma has demonstrated no difference in terms of in-hospital mortality rate. ${ }^{27}$ In addition, the analysis of the secondary outcomes of the trial showed a statistically significant reduction in time from the presentation to the end of imaging and from the presentation to the diagnosis of life-threatening injuries in the WBCT group. ${ }^{27}$ Nevertheless, several limitations might affect the conclusion being drawn from this study. One of the limitations is a percentage of $46 \%$ of the patients who were in the control group had eventually needed to undergo WBCT that is of non-immediate type, which creates an overlapping effect between the study and control group that questioned the results. ${ }^{27}$ Additionally, there was a large number of exclusions that might otherwise benefit from the use of initial WBCT. ${ }^{27}$ Although the study has many strengths and has added significant value to the existing literature, the appropriate patient selection criteria for those who might benefit from the use of WBCT during initial diagnostic work-up remains challenging.

Multiple studies reported different algorithms and criteria' for patients' selection and utilization of WBCT in trauma, which could have the potential of reducing unnecessary WBCT and their associated consequences. ${ }^{9-14}$ These studies have provided several components that can be used in the selection criteria for WBCT, which could be the base for formulating best practice guidelines. This study highlights the need for high-quality studies to accurately identify highly sensitive and specific predictors to develop a unified international guideline that has the potential to reduce the rate of negative WBCT safely.

\section{Limitations}

This study has several limitations. A major limitation of the present study is the inclusion of only patients who underwent trauma team activation. Moreover, it was a retrospective chart review and conducted at a single integrated-delivery health system in the KSA; therefore, it may have a limited generalizability to other healthcare settings. Also, the retrospective observational approach limited our identification of WBCT scans which influence the pre-scan course of management, due to the unavailability of data to answer this question in our trauma database. Our data was limited to the ED, in which we were unable to examine the influence of WBCT on neither admission management nor the in-hospital outcome. Percentages, investigations, and treatment of anaphylaxis, contrast-induced nephropathy, and incidentalomas were not recorded. Finally, the study relied solely on retrospective data collection; thus, it is subjected to the accuracy and entirety of documentation by the primary treating team. 


\section{Conclusions}

In conclusion, rapid recognition of traumatic life-threatening injuries is critical to achieve good outcomes. WBCT is undoubtedly a preferred and valuable diagnostic modality in the detection of acute traumatic injuries in polytraumatized patients. Our study showed a slightly higher rate of utilization of WBCT in the management of trauma patients as compared to similar studies. However, it is difficult to determine the exact extent of overutilization of each scan. This study demonstrates the need for appropriate selection criteria, which might reduce unnecessary scans. Still, we believe that in the correct setting with incorporating high index of suspicion, a physical examination with attention to vital signs and mental status, performing E-FAST, and dedicated X-Rays is a way to potentially reduce the use of WBCT in polytrauma patients. Based on these findings, we strongly advocate for the use of a comprehensive approach to drive WBCT decision.

\section{Patient and Public Involvement}

Patients and/or the public were not involved in the design, or conduct, or reporting or dissemination plans of this research.

\section{Abbreviations}

KSA, Kingdom of Saudi Arabia; MVC, Motor Vehicle Collision; WBCT, Whole-body Computed Tomography; IRB, Institutional Review Board; ED, Emergency Department; SPSS, Statistical Packages for Social Sciences; SD, Standard Deviation; IQR, Inter-quartile Range; CI, Confidence Interval; SBP, Systolic Blood Pressure; MAP, Mean Arterial Pressure; $\mathrm{SpO}_{2}$, oxygen saturation; SICU, Surgical Intensive Care Unit; HLOS, Hospital Length of Stay; GCS, Glasgow Coma Score; ISS, Injury Severity Score.

\section{Data Sharing Statement}

The data are available upon reasonable request to the corresponding author. These data do not include any patient-specific personal information.

\section{Ethical Approval}

The monitoring office for research and research ethics (MORRE) at Imam Abdulrahman Bin Faisal University (IAU) has granted ethical permission, which was certified with the following registration number IRB-2018-11-176. Written informed consent was waived by our institutional ethics committee due to the retrospective chart review nature of the study. Patients' data confidentiality was maintained throughout the study conduction and reporting. The study was conducted and reported in compliance with institutional and national ethics committees and with the Helsinki Declaration (as revised in 2013).

\section{Author Contributions}

All authors made substantial contributions to conception and design, acquisition of data, or analysis and interpretation of data; took part in drafting the article or revising it critically for important intellectual content; agreed on the journal to which the article will be submitted; gave final approval of the version to be published; and agree to be accountable for all aspects of the work.

\section{Funding}

This research did not receive any funding.

\section{Disclosure}

The authors declare that they have no competing interests.

\section{References}

1. Long B, April MD, Summers S, Koyfman A. Whole body CT versus selective radiological imaging strategy in trauma: an evidence-based clinical review. Am J Emerg Med. 2017;35(9):1356-1362. doi:10.1016/j.ajem.2017.03.048

2. Al-Naami MY, Arafah MA, Al-Ibrahim FS. Trauma care systems in Saudi Arabia: an agenda for action. Ann Saudi Med. 2010;30 (1):50-58. doi:10.5144/0256-4947.59374

3. DeNicola E, Aburizaize OS, Siddique A, Khwaja H, Carpenter DO. Road traffic injury as a major public health issue in the Kingdom of Saudi Arabia: a review. Front Public Health. 2016;4:215. doi:10.3389/fpubh.2016.00215

4. Huber-Wagner S, Biberthaler P, Häberle S, et al. Whole-Body CT in haemodynamically unstable severely injured patients a retrospective, multicentre study. PLoS One. 2013;8(7):e68880. doi: 10.1371 journal.pone. 0068880

5. Yoong S, Kothari R, Brooks A. Assessment of sensitivity of whole body CT for major trauma. Eur J Trauma Emerg Surg. 2019;45 (3):489-492. doi:10.1007/s00068-018-0926-7

6. Hsiao KH, Dinh MM, Mcnamara KP, et al. Whole-body computed tomography in the initial assessment of trauma patients: is there optimal criteria for patient selection? Emerg Med Australas. 2013;25(2):182-191. doi:10.1111/1742-6723.12041

7. Hinson JS, Ehmann MR, Fine DM, et al. Risk of acute kidney injury after intravenous contrast media administration. Ann Emerg Med. 2017;69(5):577-586.e4. doi:10.1016/j.annemergmed.2016.11.021

8. Aycock RD, Westafer LM, Boxen JL, Majlesi N, Schoenfeld EM, Bannuru RR. Acute kidney injury after computed tomography: a meta-analysis. Ann Emerg Med. 2018;71(1):44-53.e4. doi:10.1016/j. annemergmed.2017.06.041

9. Babaud J, Ridereau-Zins C, Bouhours G, et al. Benefit of the Vittel criteria to determine the need for whole body scanning in a severe trauma patient. Diagn Interv Imaging. 2012;93(5):371-379. doi:10.1016/j.diii.2012.02.007

10. Davies RM, Scrimshire AB, Sweetman L, Anderton MJ, Holt EM. A decision tool for whole-body CT in major trauma that safely reduces unnecessary scanning and associated radiation risks: an initial exploratory analysis. Injury. 2016;47(1):43-49. doi:10.1016/j. injury.2015.08.036 
11. Gordic S, Alkadhi H, Hodel S, et al. Whole-body CT-based imaging algorithm for multiple trauma patients: radiation dose and time to diagnosis. $\mathrm{Br} \quad J$ Radiol. 2015;88(1047):1047. doi:10.1259/bjr. 20140616

12. Wurmb TE, Frühwald P, Hopfner W, Roewer N, Brederlau J. Wholebody multislice computed tomography as the primary and sole diagnostic tool in patients with blunt trauma: searching for its appropriate indication. Am J Emerg Med. 2007;25(9):1057-1062. doi:10.1016/j. ajem.2007.03.016

13. Hong Z-J, Chen C-J, Yu J-C, et al. The evolution of computed tomography from organ-selective to whole-body scanning in managing unconscious patients with multiple trauma. A retrospective cohort study. Medicine. 2016;95(37):e4653. doi:10.1097/MD.0000 000000004653

14. Treskes K, Saltzherr TP, Luitse JSK, Beenen LFM, Goslings JC. Indications for total-body computed tomography in blunt trauma patients: a systematic review. Eur J Trauma Emerg Surg. 2017;43 (1):35-42. doi:10.1007/s00068-016-0711-4

15. Oosthuizen GV, Bruce JL, Bekker W, Shangase N, Laing GL, Clarke DL. Pan computed tomography for blunt polytrauma: are we doing too many? South African Medical Journal. 2016;106 (8):801-803. doi:10.7196/SAMJ.2016.v106i8.10376

16. Beak P, Gabbott B, Williamson M, Hing CB. Four years of experience as a major trauma centre results in no improvement in patient selection for whole-body CT scans following blunt trauma. European Journal of Orthopaedic Surgery \& Traumatology. 2020;30 (3):473-477. doi:10.1007/s00590-019-02592-3

17. Smith CM, Woolrich-Burt L, Wellings R, Costa ML. Major trauma CT scanning: the experience of a regional trauma centre in the UK. Emerg Med J. 2011;28(5):378-382. doi:10.1136/emj.2009. 076414

18. Linder F, Mani K, Juhlin C, Eklöf H. Routine whole body CT of high energy trauma patients leads to excessive radiation exposure. Scand J Trauma Resusc Emerg Med. 2016;24(1):1-7. doi:10.1186/s13049016-0199-2
19. Al Orf A, Waheed KB, Baig AA, et al. Patterns of injury detected by pan-computed tomography after road traffic accidents: retrospective review from a trauma center in Saudi Arabia. Ann Saudi Med. 2018;38(4):245-250. doi:10.5144/0256-4947.2018.245

20. Bendak S. Seat belt utilization in Saudi Arabia and its impact on road accident injuries. Accid Anal Prev. 2005;37(2):367-371. doi:10.1016/ j.aap.2004.10.007

21. Salim A. Whole body imaging in blunt multisystem trauma patients without obvious signs of injury: results of a prospective study. Arch Surg. 2006;141(5):468-475. doi:10.1001/archsurg.141.5.468

22. Griffey RT, Jeffe DB, Bailey T. Emergency physicians' attitudes and preferences regarding computed tomography, radiation exposure, and imaging decision support. Acad Emerg Med. 2014;21(7):768-777. doi:10.1111/acem. 12410

23. Gupta M, Schriger DL, Hiatt JR, et al. Selective use of computed tomography compared with routine whole body imaging in patients with blunt trauma. Ann Emerg Med. 2011;58(5):407-416.e15. doi:10.1016/j.annemergmed.2011.06.003

24. Yeguiayan JM, Yap A, Freysz M, et al. Impact of whole-body computed tomography on mortality and surgical management of severe blunt trauma. Crit Care. 2012;16:3. doi:10.1186/cc11375

25. Sierink JC, Saltzherr TP, Beenen LFM, et al. A case-matched series of immediate total-body CT scanning versus the standard radiological work-up in trauma patients. World J Surg. 2014;38(4):795-802. doi:10.1007/s00268-013-2310-4

26. Caputo ND, Stahmer C, Lim G, Shah K. Whole-body computed tomographic scanning leads to better survival as opposed to selective scanning in trauma patients: a systematic review and meta-analysis. J Trauma Acute Care Surg. 2014;77(4):534-539. doi:10.1097/ TA.0000000000000414

27. Sierink JC, Treskes K, Edwards MJR, et al. Immediate total-body CT scanning versus conventional imaging and selective CT scanning in patients with severe trauma (REACT-2): a randomised controlled trial. Lancet. 2016;388(10045):673-683. doi:10.1016/S0140-6736(16)30932-1
Open Access Emergency Medicine

\section{Publish your work in this journal}

The Open Access Emergency Medicine is an international, peerreviewed, open access journal publishing original research, reports, editorials, reviews and commentaries on all aspects of emergency medicine. The manuscript management system is completely online and includes a very quick and fair peer-review system, which is all easy to use. Visit http://www.dovepress.com/testimonials.php to read real quotes from published authors. 